\title{
Manutenção do trabalho e presença de condições crônicas em idosos comunitários: evidências da Rede Fibra-BH
}

\author{
Ongoing employment and chronic conditions \\ among community-dwelling elderly people: evidence \\ from Rede Fibra in Belo Horizonte
}

Juliana Lustosa Torres (https://orcid.org/0000-0002-3687-897X) ${ }^{1}$

Camila Menezes Sabino de Castro (https://orcid.org/0000-0002-5383-9796) ${ }^{2}$

Lygia Paccini Lustosa (https://orcid.org/0000-0002-0919-1320) ${ }^{3}$
${ }^{1}$ Departamento de Medicina Preventiva, Universidade Federal do Rio de Janeiro. Av. Horácio Macedo S/N, Ilha do Fundão. 21941-598 Rio de Janeiro RJ Brasil. jlt.ufmg@gmail.com

${ }^{2}$ Centro de Pesquisas René

Rachou, Fiocruz. Belo

Horizonte MG Brasil.

${ }^{3}$ Departamento de

Fisioterapia, UFMG. Belo

Horizonte MG Brasil.

\begin{abstract}
This cross-sectional study was based on a probabilistic sample of 597 community-dwelling elderly people living in Belo Horizonte. Theaim was to assess which chronic conditions are independently associated with ongoing employment among elderly people. It was conducted to assess the isolated effect of each one. The multivariate analysis was based on Poisson regression models with robust variance, adjusted by sex, age, schooling, retirement and chronic conditions. Arthritis (or rheumatism) was the only chronic condition with independent and statistic significant association with ongoing employment, even after adjustment for other chronic conditions: older people with medical diagnosis of arthritis have lower odds of being in the labor market (Fully adjusted Prevalence ratio $[P R]=0.54 ;$ CI 95\%: 0.35-0.85). Moreover, our results showed that gender modifies this association, with a lower propensity among females ( $P R=0.45$; CI 95\%: 0.25-0.84). Our results highlight the importance of health promotion among workers, mostly arthritis prevention and management among women.
\end{abstract}

Key words Employment, Health of the elderly, Chronic illness, Arthritis
Resumo Este estudo transversal foi conduzido em uma amostra probabilística de 597 idosos comunitários residentes em Belo Horizonte, com o objetivo de avaliar quais condições crônicas apresentam associações independentes com a permanência do idoso no mercado de trabalho, a fim de verificar a influência de cada uma isoladamente. A análise multivariada foi baseada em modelos de regressão de Poisson, com variância robusta e ajustadas por sexo, idade, escolaridade, aposentadoria e condições crônicas. A condição de saúde que apresentou associação independente e estatisticamente significante com o trabalho atual foi a artrite (ou reumatismo), mesmo após ajuste por outras condições crônicas, de modo que idosos que relataram diagnóstico médico de atrite tiveram menor propensão de permanecerem no mercado de trabalho (Razão de Prevalência [RP] ajustada = 0,54; IC 95\%: 0,35-0,85). Além disso, os resultados mostraram que o sexo modifica essa associação, sendo essa propensão menor somente entre o sexo feminino ( $R P=0,45$; IC 95\%: 0,25-0,84). Estes resultados reforçam a importância da promoção da saúde entre os trabalhadores, principalmente na prevenção e controle da artrite entre as mulheres.

Palavras-chave Emprego, Saúde do idoso, Doença crônica, Artrite 


\section{Introdução}

O rápido envelhecimento populacional no Brasil e em outros países em desenvolvimento ${ }^{1}$ gera a necessidade de mudanças no sistema de saúde para suprir as novas demandas da população. Concomitantemente, mudanças nas regras de aposentadoria vêm ocorrendo no Brasil, como, por exemplo, a mudança da aposentadoria compulsória do servidor público de 70 para 75 anos $^{2}$. Como consequência, os idosos tendem a permanecer formalmente no mercado de trabalho até idades mais avançadas, o que corrobora com dados do envelhecimento e aumento da População Economicamente Ativa (PEA) no Brasil ${ }^{3}$.

Segundo a Organização Mundial da Saúde ${ }^{4}$, a permanência do idoso no mercado de trabalho é uma das expressões do envelhecimento ativo, no entanto, constitui-se um grande desafio para a sociedade, principalmente para os países em desenvolvimento, onde o trabalho pode ser uma necessidade na velhice ${ }^{5}$. Nesse sentido, vários estudos têm sido desenvolvidos com o objetivo de compreender a escolha do idoso em permanecer trabalhando, tanto em países desenvolvidos ${ }^{6-11}$, como no Brasil ${ }^{12-17}$, e têm mostrado que um dos principais fatores associados à permanência dos idosos no mercado de trabalho é a condição de saúde.

A maioria dos estudos considera como condições a autopercepção da saúde $e^{6,10,13-15}$, ou o número de doenças crônicas ${ }^{10,13-16}$. Entretanto, é importante conhecer quais doenças crônicas influenciam negativamente na permanência do idoso no mercado de trabalho. Alguns estudos demonstraram que a artrite ${ }^{7,11}$, a depressão ${ }^{11}$ e o déficit cognitivo ${ }^{12}$ são fatores determinantes na relação envelhecimento e trabalho. Um estudo conduzido nos Estados Unidos ${ }^{7}$ mostrou que trabalhadores com idade mais avançada ( 65 anos ou mais) que apresentavam artrite tinham pior condição de saúde, independentemente da categoria de trabalho. Da mesma forma, na Austrália, demonstrou-se que homens com artrite e depressão apresentaram menor propensão de estar em um trabalho de tempo integral, ao passo que entre as mulheres essa associação não foi evidenciada ${ }^{11}$. Outro estudo conduzido em trabalhadores de uma indústria na Coreia ${ }^{9}$ encontrou uma correlação positiva entre nível cognitivo e capacidade para o trabalho, de modo que quanto melhor a capacidade para o trabalho, melhor a capacidade cognitiva.

Considerando a pouca informação a respeito da população brasileira acerca da permanência no mercado de trabalho e o efeito isolado de cada condição crônica, o objetivo deste estudo foi avaliar a associação entre a permanência do idoso no mercado de trabalho e a presença de condições crônicas, em uma amostra aleatória de idosos residentes em Belo Horizonte - MG.

\section{Métodos}

\section{Tipo de estudo}

Trata-se de um estudo transversal, no qual foram analisados dados da Rede FIBRA (Rede de Estudo da Fragilidade do Idoso).

\section{População em estudo}

A Rede FIBRA é um estudo multicêntrico, realizado em várias cidades do Brasil, dentre elas, Belo Horizonte-MG, cujo objetivo é traçar perfis de Fragilidade de Idosos brasileiros. A obtenção da amostra ocorreu segundo o método de amostragem casualizada por conglomerados por área, em duas fases, conforme os seguintes passos:

Para o cálculo da amostra de cada cidade foi estimado o tamanho amostral necessário para se ter uma proporção populacional de $50 \%$ de uma determinada característica (valor em que o tamanho amostral obtido foi o máximo possível ( $\mathrm{p}=0.50 ; \mathrm{q}=0.50$ ), conforme a população idosa de cada uma das cidades. Foi fixado o nível de significância de $5 \%$ (alfa $=5 \% ; Z=1,96)$. O erro amostral variou entre $3 \%$ e $5 \%$. A fórmula utilizada foi:

$$
n=\left\{z^{2} x\left[p \times q /(d)^{2}\right]\right\}
$$

Com base nesses cálculos, o tamanho amostral para as cidades com mais de um milhão de habitantes foi fixado em 601 idosos, para um erro de $4 \%$.

Inicialmente, selecionou-se aleatoriamente os setores censitários, definidos pelo Instituto Brasileiro de Geografia e Estatística (IBGE), a serem incluídos. Posteriormente, em cada um dos setores sorteados, de acordo com a proporção de idosos segundo dados do censo mais recente na época da coleta de dados (censo de 2000), estabeleceu-se a amostra mínima para cada setor. Regiões com maior concentração de idosos tiveram mais idosos incluídos, a fim de que fossem representativos dos idosos de Belo Horizonte. Todos os domicílios das ruas sorteadas foram visitados, sendo incluídos todos os idosos que aceitaram participar do estudo. 


\section{Coleta de dados}

Os dados foram coletados de dezembro de 2008 a dezembro de 2009, no domicílio do idoso, por entrevistadores previamente treinados. Cada domicílio foi visitado até três vezes em horários diferentes e, sempre que possível, informações sobre a presença de idosos em domicílios nos quais ninguém nos atendeu foram consideradas. $\mathrm{O}$ kit de coleta, composto por aparelho de pressão, balança (em quilos) e fita métrica (em milímetros), para a realização de medidas objetivas, foram padronizados e calibrados periodicamente.

Foram incluídos idosos com 65 anos ou mais, a fim de viabilizar a comparação desta população com achados de outros países. Os critérios de inclusão foram: pontuação superior a 17 no Mini-exame do estado mental (MEEM) ${ }^{18}$, ou seja, ausência de déficit cognitivo; ausência de sequelas graves de acidente vascular cerebral; ausência de doença de Parkinson grave; não fazer uso de cadeira de rodas e não estar acamado provisória ou definitivamente. Estes critérios foram adotados a fim de excluir idosos que não apresentavam marcha comunitária.

\section{Variáveis do estudo}

A variável dependente deste estudo foi o trabalho atual, obtido pela pergunta " $\mathrm{O}(\mathrm{A})$ senhor(a) trabalha atualmente?", com as opções de resposta "Sim" e "Não". Somente foram considerados como trabalhadores atuais aqueles que relataram que trabalhavam regularmente (cinco dias por semana), em horário parcial ou integral, formal ou informalmente e recebiam remuneração pelos serviços prestados. As variáveis independentes foram as condições de saúde [alteração de peso (IMC normal (referência), IMC elevado e IMC baixo), pressão arterial sistólica (PAS), depressão, artrite, doença do coração, diabetes e presença de fragilidade]. A alteração de peso foi definida de acordo com pontos de corte do IMC (peso/altura ${ }^{2}$ ), considerando a normalidade de 22 a $27 \mathrm{~kg} / \mathrm{m}^{2}{ }^{19}$. Valores abaixo de 22 foram classificados como IMC baixo e acima de 27 como IMC elevado; a PAS elevada foi definida com pressão arterial sistólica $\geq 140 \mathrm{mmHg}$; diagnostico médico para depressão, artrite (incluindo também artrite reumatoide e osteoartrose), doença do coração (angina e infarto) e câncer foram mensurados por meio da pergunta: "No último ano, algum médico já disse que o(a) senhor(a) tem...?". Os critérios adotados para a classificação da fragilidade foram aqueles descritos por Fried et al. ${ }^{20}$. Possíveis variáveis de confusão incluíram características sociodemográficas (sexo, idade e anos completos de escolaridade) e aposentadoria ( $\operatorname{sim} /$ não).

\section{Análise dos dados}

O teste qui-quadrado de Pearson foi utilizado para orientar a interpretação da análise univariada. As forças das associações entre trabalho atual e condições de saúde foram baseadas em razões de prevalência (RP), estimadas por meio de modelos de regressão de Poisson, com variância robusta, já que a variável resposta apresenta prevalência elevada. As variáveis de confusão foram definidas a priori, baseadas em relatos da literatura, sendo aposentadoria, sexo, idade e escolaridade. Primeiramente, foi avaliada a associação entre trabalho atual e cada uma das condições crônicas incluídas no estudo, separadamente (análise bruta). Posteriormente, no modelo 1, essas análises foram ajustadas pelas características sociodemográficas (sexo, idade e escolaridade) e aposentadoria. Para o modelo 2 (modelo final), foi acrescido o ajuste por variáveis de saúde (todas as condições crônicas). Uma vez que é descrito na literatura que o número de doenças crônicas associa-se com a condição de trabalho, todas as condições crônicas foram mantidas no modelo final. Todas as análises foram realizadas no pacote estatístico Stata versão 13.0 (Stata Corp., College Station, Estados Unidos), considerando parâmetros de amostra complexas (efeito do desenho e setor censitário).

\section{Aspectos éticos}

O estudo foi aprovado pelo Comitê de Ética e Pesquisa da Universidade Federal de Minas Gerais. Todos os participantes assinaram o termo de consentimento livre e esclarecido.

\section{Resultados}

Entre os 606 participantes da amostra da Rede FIBRA/BH, 597 (98,5\%) possuíam informações completas para todas as variáveis do estudo e foram incluídos na presente análise. A distribuição das características sociodemográficas e das condições de saúde de todos os participantes do estudo, segundo o trabalho atual, estão apresentadas na Tabela 1. A média da idade dos participantes foi de 74,3 anos (DP $\pm 6,4$ ), sendo a maioria do sexo feminino (66\%), com escolaridade inferior a 8 anos (69\%) e aposentados (74,4\%). Quanto ao 
Tabela 1. Distribuição das características entre todos os participantes do estudo e segundo a informação de estar ou não trabalhando $(\mathrm{N}=597)$ (Belo Horizonte, 2008).

\begin{tabular}{|c|c|c|c|c|}
\hline Variáveis & $\begin{array}{c}\text { Total } \\
\mathrm{N}=597\end{array}$ & \multicolumn{2}{|c|}{$\begin{array}{c}\text { Trabalho atual } \\
\text { Não Sim } \\
\mathrm{N}=491 \mathrm{~N}=106\end{array}$} & P valor ${ }^{\star}$ \\
\hline Homens & $203(34,0)$ & $158(77,8)$ & $45(22,2$ & 0,04 \\
\hline Mulheres & $394(66,0)$ & $333(84,5)$ & $61(15,5)$ & \\
\hline Média de idade (DP) & $74,3(6,4)$ & $74,6(6,5)$ & $73,2(5,8)$ & 0,04 \\
\hline \multicolumn{5}{|c|}{ Escolaridade (anos completos), n (\%) } \\
\hline$\geq 8$ & $185(31,0)$ & $158(85,4)$ & $27(14,6)$ & 0,02 \\
\hline $4-7$ & $239(40,0)$ & $149(86,1)$ & $55(23,0)$ & \\
\hline $0-3$ & $173(29,0)$ & $149(86,1)$ & $24(13,9)$ & \\
\hline Aposentado, n (\%) & $444(74,4)$ & $364(82,0)$ & $80(18,0)$ & 0,78 \\
\hline IMC normal, n (\%) & $240(40,2)$ & $192(80,0)$ & $48(20,0)$ & 0,22 \\
\hline IMC baixo & $86(14,4)$ & $76(88,4)$ & $10(11,6)$ & \\
\hline IMC elevado & $271(45,4)$ & $223(82,3)$ & $48(17,7)$ & \\
\hline PAS elevada, n (\%) & $349(58,5)$ & $283(81,1)$ & $66(18,9)$ & 0,38 \\
\hline Depressão, n (\%) & $101(16,9)$ & $90(89,1))$ & $11(10,9)$ & 0,04 \\
\hline Artrite, n (\%) & $159(26,6)$ & $144(90,6)$ & $15(9,4)$ & $<0,01$ \\
\hline Doença do coração, n (\%) & $73(12,2)$ & $66(90,4)$ & $7(9,6)$ & 0,05 \\
\hline Diabetes, n (\%) & $101(16,9)$ & $87(86,1)$ & $14(13,9)$ & 0,26 \\
\hline Fragilidade & $45(7,5)$ & $40(88,9)$ & $5(11,1)$ & 0,22 \\
\hline Freqüência (\%) & 100 & 82,2 & 17,8 & \\
\hline
\end{tabular}

trabalho atual, $17,8 \%$ informaram ter algum tipo de trabalho remunerado, sendo a maior proporção de homens $(22,2 \%)$ em relação às mulheres (15,5\%). Na análise univariada, o sexo, a média de idade, a escolaridade e a prevalência de artrite e de depressão apresentaram associações estatisticamente significantes $(\mathrm{p}<0,05)$ com o trabalho atual.

Os resultados da análise multivariada da associação entre trabalho atual e cada uma das condições de saúde estão apresentados na Tabela 2. Resultados significativos foram encontrados tanto na análise ajustada por características sociodemográficas quanto no modelo final, somente em relação à artrite (Razão de Prevalência [RP] bruta = 0,46; IC 95\%: 0,28-0,73; RP ajustada por aposentadoria, sexo, idade e escolaridade $=0,47$; IC 95\%: 0,30-0,76 e RP do modelo final = 0,54; IC 95\%: 0,35-0,85). Estes resultados demonstraram que pessoas, com 65 anos ou mais de idade, com artrite têm menor propensão a estarem trabalhando atualmente.

Adicionalmente, considerando-se que o sexo é uma variável descrita na literatura como associada à permanência de idosos no mercado de trabalho (apesar de não estar associada neste estudo no modelo final $[\mathrm{p}=0,225]$ ) e a prevalência de artrite ser maior no sexo feminino (prevalência ajustada, em nosso estudo, foi de $11,3 \%$ para o sexo feminino e $10 \%$ para o sexo masculino) realizou-se uma análise para verificar possível interação destas variáveis (Tabela 3 ). Os resultados mostraram que a artrite diminui a propensão de estar trabalhando, somente entre as mulheres ( $R P=0,45$; IC 95\%: 0,25-0,84 para o sexo feminino e $\mathrm{RP}=0,17$; IC 95\%: 0,02-1,53 para o sexo masculino).

\section{Discussão}

Os resultados do presente estudo mostraram que entre as condições de saúde estudadas, a artrite e a depressão foram as que apresentaram associação significante com o trabalho atual. Contudo, após ajustamentos por sexo, idade, escolaridade, aposentadoria e demais condições de saúde, apenas a artrite apresentou associação significante. Além disso, os resultados mostraram que há uma modificação de efeito do sexo nessa associação, sendo significante somente entre as mulheres. 
Tabela 2. Análise multivariada dos fatores associados ao trabalho atual $(\mathrm{N}=597)$ (Belo Horizonte, 2008).

\begin{tabular}{|c|c|c|}
\hline $\begin{array}{c}\text { Variáveis } \\
\text { Condição crônica de saúde vs não ter }\end{array}$ & $\begin{array}{c}\text { Modelo } 1 \\
\text { RP Ajustada } \\
\text { (IC 95\%) }\end{array}$ & $\begin{array}{c}\text { Modelo } 2 \\
\text { RP Ajustada } \\
\text { (IC 95\%) }\end{array}$ \\
\hline IMC normal & 1,00 & 1,00 \\
\hline IMC baixo & $0,59(0,28-1,23)$ & $0,58(0,28-1,19)$ \\
\hline IMC elevado & $0,92(0,60-1,41)$ & $0,99(0,65-1,51)$ \\
\hline PAS elevada & $1,19(0,83-1,70)$ & $1,13(0,80-1,60)$ \\
\hline Depressão & $0,61(0,30-1,23)$ & $0,67(0,33-1,35)$ \\
\hline Artrite & $0,47(0,30-0,76)^{\star *}$ & $0,54(0,35-0,85)^{*}$ \\
\hline Doença do coração & $0,52(0,22-1,23)$ & $0,61(0,25-1,51)$ \\
\hline Diabetes & $0,72(0,47-1,10)$ & $0,80(0,52-1,23)$ \\
\hline Fragilidade & $0,69(0,26-1,86)$ & $0,85(0,31-2,36)$ \\
\hline
\end{tabular}

Tabela 3. Análise multivariada entre trabalho atual, sexo e presença de artrite $(\mathrm{N}=597)$ (Belo Horizonte, 2008).

\begin{tabular}{lcc}
\hline & Prevalência Ajustada (\%) & RP Ajustada (IC 95\%) \\
\hline Sexo masculino e ausência de artrite & 25,2 & 1,00 \\
Sexo feminino e ausência de artrite & 17,1 & $0,68(0,42-1,09)$ \\
Sexo masculino e presença de artrite & 4,2 & $0,17(0,02-1,53)$ \\
Sexo feminino e presença de artrite & 11,4 & $0,45(0,25-0,84)^{*}$ \\
\hline
\end{tabular}

IC95\%: intervalo de $95 \%$ de confiança; ${ }^{\star} \mathrm{p}<0,05$. Prevalência e RP ajustados pelas características sociodemográficas (faixa etária e escolaridade), aposentadoria e por condições crônicas de saúde (sobrepeso, Pressão arterial sistólica elevada, depressão, doença do coração e diabetes); RP: razão de prevalência estimada pelo modelo de regressão de poisson.

Corroborando com estudos anteriores, a participação dos homens idosos no mercado de trabalho é maior que a das mulheres. Estudos conduzidos em dez regiões metropolitanas brasileiras ${ }^{13,14}$ estimaram que a prevalência dos idosos, com 65 anos ou mais, no mercado de trabalho foi de $26,9 \%$ entre os homens ${ }^{14}$ e $8,9 \%$ entre as mulheres ${ }^{13}$.

As diferenças nas taxas de participação no trabalho entre homens e mulheres com idade mais avançada ocorrem principalmente nos países em desenvolvimento, o que pode ser devido às situações que as mulheres enfrentaram ao longo da vida, como baixo nível educacional e desenvolvimento de habilidades, menores oportunidades econômicas e exposição à violência em relação ao gênero ${ }^{21}$.

Além disso, os idosos que permaneceram no mercado de trabalho pertenciam às faixas etárias mais jovens, sendo que dos idosos que trabalhavam 32,4\% tinham entre 65 e 69 anos, e mais da metade $(63 \%)$ possuíam até 74 anos de idade (dados não mostrados). Essa tendência de diminuição da atividade laboral com a idade é identificada em todo o Brasil. Segundo Rosa ${ }^{22}$, no ano de 2012, idosos brasileiros na faixa etária de 60-69 anos eram mais ativos no mercado de trabalho, representando cerca de $90 \%$ contra $8 \%$ dos idosos com idade entre 70-79 anos e, apenas $1 \%$ dos idosos com 80 anos e mais.

A associação entre presença de artrite e menor participação no trabalho entre idosos, encontrada neste estudo, está de acordo com o observado em demais estudos brasileiros ${ }^{13-15}$ e nos Estados Unidos ${ }^{7}$. A artrite é, dentre as doenças reumáticas, a mais prevalente na população idosa, sendo a maior incidência, prevalência e gravidade entre as mulheres. É uma condição crônica-degenerativa associada à dor, rigidez articular, deformidade e incapacidade funcional, afetando o indivíduo nas dimensões físicas, emocionais, funcionais e sociais ${ }^{23}$. Segundo Schofield et al. ${ }^{24}$, a artrite afeta em grande medida a capacidade de trabalhar em homens e mulheres, devido à dor e à incapacidade. Entretanto, resultados conflitantes foram encontrados quando comparado a um estudo australiano ${ }^{11}$, no qual a associação encontrada entre presença de artrite e menor par- 
ticipação no trabalho foi somente entre homens. Essas diferenças podem ser devido à diferente categorização da variável trabalho, visto que no estudo australiano o trabalho foi definido por três categorias (trabalho em tempo integral, trabalho em meio período e estar fora do mercado de trabalho), enquanto que no presente estudo as categorias trabalho em tempo integral/meio período foram consideradas em uma categoria única.

Em idades mais jovens (até 64 anos), a literatura mostra que as principais variáveis associadas a não trabalhar, em portadores de artrite, são principalmente menor escolaridade e dor intensa, que pode ser devido à diminuição da força, limitação da amplitude de movimento e deformidades ${ }^{25}$. Entre mulheres, não apresentar cônjuge e a presença de incapacidade severa devido à artrite também desempenham papel importante ${ }^{26}$. Além disso, um estudo mais recente encontrou que a incapacidade para o trabalho está associada diretamente com a severidade da incapacidade funcional ${ }^{27}$. Nos Estados Unidos, por exemplo, além da prevalência de artrite ser maior entre as mulheres, $23 \%$ apresenta alguma limitação de atividade relacionada a ela ${ }^{28}$, contra $14,5 \%$ entre os homens.

Uma hipótese seria que a maior incapacidade nas mulheres, consequente a artrite, as impede de continuarem no mercado de trabalho. Os sintomas da doença, o seu controle e as condições de trabalho e suas adaptações desempenham um papel importante para a manutenção do trabalho em portadores de artrite $^{29}$ e, como entre as mulheres a prevalência e a incapacidade subsequente são maiores, manter-se trabalhando torna-se mais difícil. Uma segunda hipótese seria que, entre os homens, o significado de trabalho e as condições socioeconômicas ${ }^{29}$ são mais importantes, visto que, historicamente, são os provedores da casa. Assim, e por apresentarem menor prevalência de incapacidade após a artrite estão mais aptos a permanecerem trabalhando. Uma terceira hipótese seria que as atividades desempenhadas no mercado de trabalho são diferenciadas entre homens e mulheres. Neste contexto, segundo o Banco Mundial ${ }^{30}$, em quase todos os países, as mulheres idosas têm maior probabilidade de participar de atividades de baixa produtividade, ter um emprego familiar assalariado ou não remunerado, participar do mercado de trabalho informal, gerenciar empresas menores e se concentrar nos setores menos lucrativos. As três hipóteses não são mutuamente excludentes.

Diferentemente de outros estudos ${ }^{9,11}$, a depressão foi associada com a permanência dos idosos no trabalho neste estudo somente na análise univariada. Neste caso, uma vez que um dos critérios de inclusão do estudo FIBRA foi o escore do MEEM acima de 17 pontos, isto pode ter sido uma limitação e a interpretação desta ausência de associação entre déficit cognitivo e trabalho deve ser vista com cautela. Um estudo com pessoas em idade ativa encontrou que aqueles com depressão eram mais propensos a estar desempregados, terem mudado de emprego recentemente e receber menores salários ${ }^{31}$. Em idosos jovens (60-64 anos), a presença de depressão foi associada com a diminuição da propensão de trabalhar em um emprego de tempo integral entre os homens ${ }^{11}$. Desta forma, sugere-se a realização de novos estudos considerando possíveis déficits cognitivos.

Assim como a maioria dos estudos epidemiológicos, este estudo apresenta vantagens e limitações. A principal vantagem é o rigor na seleção da amostra e na metodologia da pesquisa, garantindo a sua validade interna e permitindo generalização dos resultados para idosos comunitários residentes em Belo Horizonte. No entanto, ao se discutir os resultados do presente estudo deve-se levar em consideração o desenho transversal, que impede de analisar relações temporais entre as variáveis e a interpretação da associação entre artrite e trabalho atual como possível causalidade reversa, uma vez que não se pode concluir se idosos com melhores condições de saúde têm maior probabilidade de trabalhar ou se os idosos que trabalham possuem melhores condições de saúde. Outras limitações são a ocorrência do viés do trabalhador sadio, no qual se observa que parte dos trabalhadores pode ser excluída do mercado de trabalho, devido à sintomatologia das doenças crônicas, $\mathrm{o}$ que permite que indivíduos mais saudáveis apresentam maior probabilidade tanto de ingressar na força de trabalho, quanto de permanecerem trabalhando. Bem como o viés de fonte única, já que a variável trabalho, assim como algumas variáveis foram avaliadas através de autorrelato. Entretanto, considerou-se o diagnóstico médico para as doenças autorrelatadas. Ainda, na variável aposentadoria, não foi considerada a diferença entre trabalhadores aposentados por invalidez, por tempo de serviço e motivação pessoal. Essas diferentes categorias poderiam associar-se à manutenção do trabalho de diferentes maneiras.

Concluindo, este estudo sugere que a artrite entre as mulheres pode gerar barreiras para a continuidade da participação das idosas no mercado de trabalho. Com as recentes mudanças no mercado de trabalho e o aumento da expectativa de vida, há maior necessidade de promoção da 
saúde entre os trabalhadores, principalmente na prevenção e controle da artrite. Medidas como ginástica laboral e adaptações no ambiente de trabalho tornam-se fundamentais para a manu- tenção do idoso no mercado de trabalho. Além disso, deve-se considerar diferenças entre sexos e como os diferentes aspectos da saúde física atuam nesses trabalhadores.

\section{Colaboradores}

JL Torres contribuiu para a conceituação do estudo e seu desenho; JL Torres e LP Lustosa contribuíram no recrutamento de sujeitos e/ou dados; JL Torres, CMS Castro e LP Lustosa contribuíram para a análise e interpretação dos dados; e JL Torres, CMS Castro e LP Lustosa contribuíram para a preparação do manuscrito.

\section{Agradecimentos}

Os autores gostariam de agradecer o financiamento proporcionado pelo Conselho Nacional de Desenvolvimento Científico e Tecnológico (CNPq), pela Fundação de Amparo à Pesquisa de Minas Gerais (FAPEMIG) e pela Coordenação de Aperfeiçoamento de Pessoal de Nível Superior (CAPES).

\section{Referências}

1. US National Institute of Aging, World Health Organization (WHO). Global Health and Aging. Washington: NIH; 2011. NIH Publication No.: 11-7737.

2. Brasil. Lei complementar $\mathrm{n}^{\circ} 152$, de 3 de dezembro de 2015. Dispõe sobre a aposentadoria compulsória por idade, com proventos proporcionais, nos termos do inciso II do $\$ 1^{\circ}$ do art. 40 da Constituição Federal. Diário Oficial da União 2015; 4 dez.

3. Instituto de Pesquisa Econômica Aplicada (IPEA). Mercado de trabalho: conjuntura e análise. Brasília: IPEA; 2013.

4. World Health Organization (WHO). Active ageing: a policy framework. 2002. [acessado 2016 Set 5]. Disponível em: http://apps.who.int/iris/bitstream/10665/67215/1/WHO_NMH_NPH_02.8.pdf

5. Programa das Nações Unidas para o Desenvolvimento (PNUD). Relatório do desenvolvimento humano 2015: O trabalho como motor do desenvolvimento humano. [acessado 2016 Set 5]. Disponível em: http:// www. undp.org

6. Mazzonna F. The long lasting effects of education on old age health: evidence of gender differences. Soc Sci Med 2014; 101:129-138.

7. Caban-Martinez AJ, Lee DJ, Fleming LE, Tancredi DJ, Arheart KL, Leblanc WG, McCollister KE, Christ SL, Louie GH, Muenning PA. Arthritis, occupational class, and the aging US workforce. Am J Public Health 2011; 101(9):1729-1734.

8. Ilmarinem J; Finnish Institute of Occupational Health; Ministry of Social Affairs and Health. Age information through research: maintaining work ability. In: Ilmarinem J; Finnish Institute of Occupational Health; Ministry of Social Affairs and Health, editors. Towards a longer worklife! Ageing and the quality of worklife in the European Union. Helsinki: Finnish Institute of Occupational Health; 2005. p. 132-148.

9. Chung J, Park J, Cho M, Park Y, Kim D, Yang D, Yang Y. A study on the relationships between age, work experience, cognition, and work ability in older employees working in heavy industry. J Phys Ther Sci 2015; 27(1):155-157. 
10. Majeed T, Forder P, Mishra G, Byles J. Women, Work, and Illness: A Longitudinal Analysis of Workforce Participation Patterns for Women Beyond Middle Age. $J$ Womens Health (Larchmt) 2015; 24(6):455-465.

11. Majeed T, Forder PM, Mishra G, Kendig H, Byles JE. Exploring Workforce Participation Patterns and Chronic Diseases Among Middle-Aged Australian Men and Women Over the Life Course. J Aging Health 2017; 29(2):343-361.

12. Camarano AA, Kanso S, Mello JL, Pasinato MT. A oferta de força de trabalho brasileira: tendências e perspectivas. In: Tafner P, organizador. Brasil: o estado de uma nação - mercado de trabalho, emprego e informalidade. Rio de Janeiro: Instituto de Pesquisa Econômica Aplicada; 2006. p. 69-118.

13. Giatti L, Barreto SM. Trabalho feminino e saúde na terceira idade. Cien Saude Colet 2002; 7(4):825-39.

14. Giatti L, Barreto SM. Saúde, trabalho e envelhecimento no Brasil. Cad Saude Publica 2003; 19(3):759-771.

15. Pérez ER, Wajnman S, Oliveira AMHC. Análise dos determinantes da participação no mercado de trabalho dos idosos em São Paulo. Rev Bras Estud Popul 2006; 23(2):269-286.

16. Castro CMS, Mambrini JVM, Sampaio RF, Macinko J, Lima-Costa MF. Aspectos sociodemográficos e de saúde associados ao trabalho remunerado em adultos (50-69 anos) na Região Metropolitana de Belo Horizonte, Minas Gerais, Brasil. Cad Saude Publica 2015; 31(8):1775-1787.

17. Godinho MR, Greco RM, Teixeira MTB, Teixeira LR, Guerra MR, Chaoubah A. Work ability and associated factors of Brazilian technical-administrative workers in education. BMC Res Notes 2016; 9(1):1-10.

18. Brucki SMD, Nitrini R, Caramelli P, Bertolucci PHF. Sugestões para o uso do mini-exame do estado mental no Brasil. Arq. Neuro-Psiquiatr 2003; 61(3B):777-781.

19. Nutrition Screening Initiative. Incorporating nutrition screening and interventions into medical practice. A monograph for physicians. Washington: American Academy of Family Physicians, The American Dietetic Association, National Council on Aging Inc; 1994.

20. Fried LP, Tangen CM, Walston J, Newman AB, Hirsch C, Gottidiener J, Seeman T, Tracy R, Kop WJ, Burke G, McBurnie MA. Frailty in older adults: evidence for a phenotype. J Gerontol A Biol Sci Med Sci 2001; 56(3):M146-56.

21. World Bank (WB). World Bank Group Gender \& Development. Washington: WB; 2014. [acessado 2016 Ago 16]. Disponível em: www.worldbank.org/gender

22. Rosa SS. O perfil do idoso no mercado de trabalho brasileiro: análise das PNADs 2002 e 2012 [dissertação]. Rio Grande: FURG; 2015.
23. Santos MLAS, Gomes WF, Queiroz BZ, Rosa NMBR, Pereira DS, Dias JMD, Pereira LSM. Desempenho muscular, dor, rigidez e funcionalidade de idosas com osteoartrite de joelho. Acta Ortop Bras 2011; 19(4):193197.

24. Schofield D.J, Shrestha RN, Percival R, Passey ME, Callander EJ, Kelly SJ. The personal and national costs of lost labour force participation due to arthritis: An economic study. BMC Public Health 2013; 13(1):188.

25. Andrade JA, Brandão MB, Pinto MRC, Lanna CCD. Factors Associated With Activity Limitations in People With Rheumatoid Arthritis. American Journal of Occupational Therapy 2016; 70(4):1-7.

26. Kaptein SA, Gignac MA, Badley EM. Differences in the workforce experiences of women and men with arthritis disability: a population health perspective. Arthritis Rheum 2009; 61(5):605-613.

27. Bertin P, Fagnani F, Duburcq A, Woronoff AS, Chauvin P, Cukierman G, Tropé-Chirol S, Joubert JM, Kobelt G. Impact of rheumatoid arthritis on career progression, productivity, and employability: The PRET Study. Joint Bone Spine 2016; 83(1):47-52.

28. Hootman JM, Helmick CH. Projections of US prevalence of arthritis and associated activity limitations. Arthrits \& Rheumathology 2005; 54(1):226-229.

29. Hoving JL, van Zwieten MC, van der Meer M, Sluiter JK, Frings-Dresen MH. Work participation and arthritis: a systematic overview of challenges, adaptations and opportunities for interventions. Rheumatology (Oxford) 2013; 52(7):1254-1264.

30. Banco Mundial (BM). Relatório sobre o desenvolvimento mundial de Igualdade de gênero e desenvolvimento. Washington: BM; 2012.

31. Wang X, Guo J, Zhang X, Qu Z, Tian D, Ma S. The effects of depression and chronic diseases on the work outcomes of employees: a prospective study in Northwest China. Public Health 2014; 128(8):734-742.

Artigo apresentado em 08/02/2017

Aprovado em 01/08/2017

Versão final apresentada em 03/08/2017 